\title{
Eyeing Innocent Souls: The Trajectory Of Domestic Violence And Children's Education In Botswana
}

\author{
Mavis B. Mhlauli \\ University of Botswana, Department of Primary Education. \\ Private Bag 00702, Gaborone, Botswana \\ End Salani \\ University of Botswana, Department of Primary Education. \\ Private Bag 00702, Gaborone, Botswana \\ Keinyatse Kgosidialwa \\ University of Botswana, Department of Primary Education. \\ Private Bag 00702, Gaborone, Botswana
}

\begin{abstract}
The purpose of this paper is to interrogate the effects of domestic violence among children in Botswana. Domestic violence is a global problem as it affects people from both poor and stable economies. Research has shown that there is a link between domestic violence and child abuse. This link has been shown in and confirmed by various studies conducted in a number of countries including China, South Africa, Colombia, India, Egypt, Philippines and Mexico (Unicef, 2005). Botswana like many countries of the world has a challenge of protecting children from being victims of domestic violence. Most violence in Botswana is reported to occur within intimate relationships which takes the form of emotional, physical and psychological abuse and mostly occurs among women. It has also been observed that domestic violence is common mostly in male dominated cultures which are either patriarchal or patrilineal and often justified by their customs and traditions, and condoned by law (Kanchipuntu and Mwale, 2016). This violence is said to affect children's lives as it may result in emotional trauma, physical and psychological barriers in schooling and educational experience in general (Lloyd, 2018). Paradoxically, children find themselves entangled in domestic violence with very little knowledge of what steps to take to end the form of abuse they experience. Governments, education systems and schools in particular have a role to play to mitigate the situation that children find themselves in hence the suggested strategies. More research needs to be undertaken to develop ways of dealing with domestic violence in schools which are culturally sensitive..
\end{abstract}

Keywords: Domestic violence, children, education, schooling, Early Childhood Education, Botswana, primary education, adolescents, victims.

\section{INTRODUCTION}

"I am a teacher in a private school in a village near Gaborone. I teach a standard 2 class and I have an 8 year old pupil who has been raped by a 47 year old man. As a teacher, I feel I have a responsibility to help this child. The school-head has refused to assist because the incident happened outside the school premises. I have organized counseling sessions for this child. I have to go and pick her up every morning for counseling sessions and back with the little money that I have. Today I had to walk from the station to this place in order to ask for transport money from Prof..." (Anonymous Teacher, Gaborone, Botswana) 
The above quote sets the tone of this paper as posits the pervasiveness of domestic violence among school going children and how it affects them and those who care for them. Domestic violence is one of the most common ills that have permeated the fabric of every society in the world and usually goes by unnoticed. In some societies domestic violence is embedded in the cultural practices to the extent that it is regarded as a norm. Even though laws that relate to gender-based violence such as the domestic violence act, the penal code and the criminal procedure are in place, in countries like Botswana, domestic violence at times is seen as part of culture, and there have been instances where couples have reported cases of domestic violence to the police and have been turned away and asked to resolve the issue at home. This indicates the extent to which domestic violence is sometimes not taken seriously in countries like Botswana, and many non-governmental organizations in the country have blamed the escalation of murder/ suicide cases commonly known as "passion killings" to the ignorance of the legal system in punishing acts of domestic violence.

According to Carter, Weithorn \& Behrman (1999) there is research evidence that estimates that there are between 3.3 million and 10 million children in the United States exposed to domestic violence each year and that this exposure can have significant negative effects on children's behavioral, emotional, social and cognitive development. This paper discusses the effects of domestic violence on children and will draw examples from the United States, Botswana and Nigeria where relevant. It begins by defining domestic violence, types of domestic violence, its causes, effects and preventative measures commonly used in schools.

\section{UNDERSTANDING DOMESTIC VIOLENCE}

Domestic violence is one of the concepts that has gained a lot of attention and is referred to in different settings in different ways. Some refer to it as family violence while others refer to it as intimate partner violence (IPV) or wife battering as well as spouse abuse. Another term found in the literature is inter-parental violence (Lloyd, 2018) and such terms are usually used interchangeably. For purposes of consistency, this paper will use domestic violence as it is the most commonly used term. The American Bar Association (1994) states that; "domestic violence or "battering" refers to the use of physical force, or threat of such force , against a current or former partner in an intimate relationship, resulting in fear and emotional and/or physical suffering"(p.1). The definition above concurs with that of Fantuzzo \& Mohr (1999) that domestic violence typically refers to violence between adult intimate partners. They further opine that domestic violence defined from a clinical perspective refers to a pattern of assaultive and coercive behaviors including physical, psychological and sexual attacks as well as economic coercion that adults and adolescents use against each other. From these definitions it is clear that domestic violence involves acts of force, physical harm, sexual abuse and threat of physical harm. Domestic violence has become a social issue and does not have any boundaries; it cuts across cultures, gender, ethnicity, race, religion and economic lines.

\section{Types of domestic violence}

There are various types of domestic violence and these are sometimes referred to as partner violence. Johnson and Ferraro (2000) have identified four major patterns of partner violence which are; Common Couple Violence (CCV), Intimate Terrorism (IT), Violent Resistance (VR) and Mutual Violent Control (MVC). According to Johnson \& Ferraro, CCV is the type of violence that is not connected to the general pattern of control. It is said to arise where partners have a specific argument and lash out physically at one another. Johnson further asserts that empirical research have shown that CCV has lower per couple frequency, is not likely to escalate over time, does not involve severe violence and is more likely to be mutual. In my view, this type of violence is common where people have built in anger and have unresolved issues. Instead of talking about issues of concern in their relationship they begin to shout at one another. After 
shouting at each other the issue is believed to have been resolved, it is usually temporary. They further contend that CCV is quite the opposite of Intimate Terrorism (IT) where the violence is motivated by a wish to exert general control. This is the type of violence that feminist researchers contend that it is part of a system of coercive controls over women (Kanchiputu and Mwale, 2016, Anderson, 1997).

This type of violence known as Intimate terrorism (IT) has a tendency to escalate over time and is likely to involve serious injury. The control mechanism of IT often involves emotional abuse. This is the type of abuse is common among African societies because of the cultural expectations and honor given to men. Okereke (2002) refers to this type of violence as common in societies like Nigeria where cultural values, norms and beliefs among others give men greater power and control over their spouses. For instance; if a man is married, culturally, the wife is not supposed to ask him where he is from when he did not sleep at home. Asking a man questions is culturally seen as disrespectful and men take advantage of that and abuse women. According to Johnson and Ferraro (200) this type of violence is also common among lesbian relationships and argues that emotional abuse is not the sole prerogative of men.

Another type of partner violence according to Johnson \& Ferraro (1999) is that of Violent Resistance (VR). They further indicate that there is very little evidence of research on VR, however, this is the type of violence where battered women act in defense from their partners. People always relate it to the type of violence where women usually kill their partners as a way of fighting back. Research indicates that women who fight back clearly exhibit warning signs of leaving their abusive relationships. This type of violence is usually perpetrated by women. The authors call for further research on the nature and incidents of VR in partner violence.

The last but not least type of violence discussed here is Mutual Violence Control (MVC). Johnson \& Ferraro cite Johnson $(1999,2000$ a) as having identified this type of violence as one where both partners are controlling and violent. This situation, they contend is viewed as two intimate terrorists battering for control. There is very little research as well that thoroughly explains why both partners in a relationship are violent. However, this situation is not very common even though it exists.

\section{Causes of domestic violence}

Literature reveals that domestic violence is a health problem and it is often very difficult to identify its underlying causes. Experts on the field are not agreed on what really causes domestic violence, however, for it to be prevented and managed there is a need to identify the causes of this sinful act. Many children worldwide experience or are exposed to domestic violence every day, minute and second, yet it always appears to be invisible and not talked about. Various authors have argued that domestic violence is related to poverty, race and any form of prejudice and that wherever there is domestic violence there is child abuse( Lloyd, 2018: Kanchiputu and Mwale, 2016). Anderson (1997) asserts that the cause of domestic violence from a sociological perspective is often characterized by controversy because it centers on issues of gender. He argues that the debate centers on the relative importance of gender and power as the ultimate route cause of intimate violence. This notion is in tandem with the idea by Johnson where he makes a distinction between different types of violence and this is more related to Intimate Terrorism (IT) where the objective is to exert control and power over a partner. This is further emphasized by Anderson (1997) that:

Drawing primarily on victimization research that demonstrates the severe and repetitive nature of male violence, feminist researchers contend that violence is part of a system of coercive controls through which men societal dominance over women (p.655). 
The above assertion clearly demonstrates the cause of domestic violence from a feminist and sociological perspective. These experts believe that domestic violence is a result of the patriarchal order in trying to maintain its hegemony within the society (Republic of Botswana, 2012). Sociologists further opine that national survey techniques have shown that there is a strong relationship between domestic violence and age, cohabiting partners, unemployment and socio economic status that suggest that other characteristics of the social structure may engender violence. From these anecdotes it is possible to argue that indeed the issue of domestic violence is complex and its causes may be attributed to a wide range of issues that are not easily understood.

\section{Theories of domestic violence}

In an attempt to try and understand the causes of domestic violence, Wolfe \& Jaffe (1999) came up with five theories that explain the causes of domestic violence. These theories include; biological theory, individual psychopathology, couple and family interactions theory, social learning and development theory and societal structure theory. These theories will be discussed below in the order that they appear in this paper.

Wolfe \& Jaffe's (1999) view is that biological theory is biological and organic and can be explained by genetics, biochemistry and changes in brain development due to trauma. This theory would fall under the intra-individual theory and encompasses not only biological but neurological factors (Okereke, 2002). Researchers in this camp have linked the trauma in early exposure to chronic violence to changes in a child's brain functioning that lead to violent behavior as an adult.

They believe that some abusive men with abusive histories tend to have a history of head injuries that affected their ability to solve problems and control impulsivity. The implication is that once these men are confronted with a problem they resort to domestic violence since they cannot think due to the injuries incurred in early life. According to this theory it is like men are the only people who engage in domestic violence, which is not always the case. There are instances where women are also perpetrators of violence. It is quite difficult to understand this theory because it appears to hold that violence is biological and not a learned behavior.

The individual psychopathology theory asserts that domestic violence is rooted in an individual dysfunctional personality structures which are more likely to be biological factors learned and shaped by early childhood experiences (Wolfe and Jaffe, 1999). According to Wolfe and Jaffe (1999) research in this area includes studies of male batterers, showing that witnessing domestic violence or being a victim of abuse undermines one's ability to trust and regulate emotions and results in hostile, dependent, insecure individuals with little ability to develop healthy relationships. Similar research has also shown that male batterers are more likely than non-batterers to perform poorly on mental health tests. This theory tends to imply that people who are exposed to violence in their early childhood have the likelihood of becoming violent as well because they tend to think that violence is the solution to every problem. This theory tends to explain what happens in everyday life situations where violence is prevalent. I have also observed that children exposed to violence tend to be violent as well because that is what they grew up seeing and believed to be what life is all about.

The other theory is that of couple and family interactions theory. This theory according to Wolfe and Jaffe "suggests that domestic violence is rooted in the faulty interactions of a couple and family system, and that an individual's violent behavior cannot be addressed without understanding the context, characteristics, and dynamics of the familial relationship" (p.134). This theory, therefore, sees interactions within the family as the core cause for domestic 
violence. It acknowledges the extent to which the role of the environment one lives in has an influence over their behavior and that faulty interactions will result in domestic violence. Okereke (2002) would refer to this theory as socio-psychological theory which posits that; "the family serves as a training ground for violence by providing both exemplars for imitation and role modeling and contingencies of reinforcement and punishment that often unintentionally encourage violence" (p. 42). This view holds that children who witness violent aggression by one spouse against the other will grow up and react to their children or spouses in the same manner thereby perpetuating the cycle of violence.

The social learning and development theory suggests that domestic violence is a learned behavior that is modeled, rewarded, and supported by families or the broader culture. According to this view abusive behavior can be learned by direct experience or by observing the behaviors of others (Okereke, 2002). Children exposed to violence according to this theory learn that aggression is appropriate to resolve conflicts, especially within the context of intimate relationship. Research has shown that batterers are much more likely to have had violent fathers than non-batterers. Wolfe and Jaffe (1999) further contend that developmental research has shown that early intervention with children from violent households may restore normal developmental processes such as empathy and self-control, and minimize the risk of further harm caused by exposure to abusive adult models. This theory reinforces the notion that children learn better when they see and engage with their environment. It clearly shows that experience is the best teacher, when they see their parents' fighting; they learn the behavior and model it in return. As a result of this exposure to domestic violence they end up being violent themselves.

The last theory according to Wolfe and Jaffe is societal structure theory. According to this perspective, domestic violence is caused by an underlying power imbalance that can be understood only by examining society as a whole. This theory blames patriarchy or male domination over women and children through physical, economic, and political control. The authors argue that domestic violence reflects women's inequality in the culture and the reinforcement of this reality by various institutions. This notion of gender inequality is supported by Anderson (1997) that;

Gender theory proposes that violence is a resource for constructing masculinity, and thus the use of violence will have different meanings for men women. Additionally, gender theory proposes that domestic violence will be affected by social processes that support men's societal dominance, such as cultural support for couplings in which men have a greater resources than their female partners (p.658).

From a gender perspective it is clear that domestic violence is caused by socio-demographic factors, status incompatibilities, relative resources and gender perspectives. Their argument is that in investigating domestic violence researchers have often ignored the gender dynamics which are very important in understanding domestic violence. To them domestic violence is caused by patriarchy and the reinforcement of societal inequalities and culture.

According to Wolfe \&Jaffe (1999) despite the divergent views postulated by these theories on the underlying causes of domestic violence, there are some zones of commonality in these theories and they are, that;

1. domestic violence has been ignored as a major social problem until recently and remains poorly understood.

2. domestic violence is a complex problem impacted by multiple variables

3. childhood trauma, either through exposure to violence or some other trauma, influences the likelihood of domestic violence 
4. as long as domestic violence is condoned as accepted behavior by public attitudes and institutions, there is little chance of preventing it (p.135).

These theories are in agreement with Pepler, Catallo \& Moore (2000) that, children growing up in violent homes have numerous potential lessons that result from regular exposure to family violence. Such children may learn that it is acceptable to be abusive and that violence is an effective way to get what you want. They may learn that violence is sometimes justified particularly if you are angry at someone. They may also learn about traditional power imbalances between men and women (Kanchiputu and Mwale, 2016). Domestic violence is an intricate and problematic issue that needs to be interrogated further in order to understand it. It is still not clear as to what really causes it, however, it appears it is a result of multiple factors. Further inquiry is needed in the area of domestic violence because it would be very problematic to try and address it if there is no agreement on its underlying causes.

\section{What does Research say?}

\section{EFFECTS OF DOMESTIC VIOLENCE ON CHILDREN}

From the studies undertaken in various parts of the world, there is evidence of documented data that points to fact that domestic violence is a major societal problem worldwide. Researchers agree that there are millions of children around the world who are exposed to domestic violence even though they are not agreed on the specific number of children affected by this pandemic. Carter, Weithorn \& Behrman (1999) opine that the most cited figures of children exposed to domestic violence is estimated between 3.3 million and 10 million. In a study carried out by Fantuzzo \& Mohr (1999) where they examined data relevant to children's exposure to domestic violence in five cities in the United States (US) found that in all the cities children witnessed domestic violence at an alarming rate. For example in Milwaukee, Wisconsin 81\% of the households in Spousal Assault Replication Program (SARP) had children present. It was also found that children under the age five were more likely to be present in the homes in which domestic violence occurred. According to Humphreys (2006) up to 86\% of children are either in the same or adjoining rooms during an incident of domestic violence. The author further alludes to the fact that the first national prevalence study of 2869 young adults indicated that $26 \%$ had witnessed violence and at least for 5\% the violence was frequent and ongoing and argues that this suggests a high number of children living with violence and abuse if calculated on an annual basis. National estimates of children exposed to domestic violence indicate that there are about 240,000-963, 000 in UK, 339, 000-2.7 million in USA, 500,00-1.3 million and a total of 34.9-38.2 million in Sub-Saharan Africa (Unicef, 2005) of which Botswana is a part.

However, in Botswana domestic violence appears to be relatively common. According to Maundeni (2000) very little work has been done on children's exposure to domestic violence in Botswana. He further contends that;

The paucity of literature on children and family violence is striking, taking into account findings of previous studies that show that violence against women is common -and that in 1991, 43\% of Botswana population was the ages between 0-14 (cf. Lesetedi \& Ngcongco, 1995). Domestic violence is so widespread that many children will have lived with it (p.165).

From Maundeni's assertion, it is clear that domestic violence is prevalent among Batswana and argues that this is related to the wider attitudes and cultural practices of Batswana. According to Setswana culture, moderate beating of wives is acceptable as the husband is regarded as the head of the family and the wife is seen as part of his belongings. The use of corporal punishment in schools is acceptable as well, as a way of moderating behavior (Maundeni, 
2000). In a study carried out among children in Botswana by Maundeni, results revealed that out of 25 children, 19 (75\%) reported that they witnessed violence towards their mothers prior to their parents' separation. Some of the children about 7 out of 19 recalled vividly the incidents where physical violence imposed on their mothers was severe and life threatening. He further argues that, not only do children witness domestic violence but are victims as well. The results from this study corroborate with those of a study carried out in Nigeria by Okereke (2002) which concluded that physical abuse is very rampant in Nigeria and that women are the primary victims. He attributes domestic violence to a number of factors among them the highly patriarchal nature of Nigerian society which encourages men to dominate, control and even exploit women and cultural norms that subjugate women to the authority of their men. $\mathrm{He}$ further argues that domestic abuse in Nigeria is exacerbated by the cultural practices where women are married at a tender age and their spouses are expected to pay bride price. This to him is the major cause for domestic violence in Nigeria since men feel that they own their wives because they paid the bride price for them and can do as they wish with them.

The perception held in Africa in general about domestic violence is very different in that it is culturally acceptable for as long as it is not excessive. The question is how do they measure excessiveness? For instance in Southern Africa, in countries like Botswana, Namibia, Mozambique, Swaziland , Zambia and Zimbabwe domestic violence is increasing at an alarming rate yet women are reluctant to report violence in the family to the extended family, police or other legal authorities (Maundeni; 2001). It is argued that these women know that they have to report such cases but do not because they know that they will not get help. The African culture that has subordinated women to men and even given men the right to chastise their wives has exacerbated the situation and encouraged them not to report, a situation that has also been observed in Nigeria, West Africa. This is one of the problems that even lead to the difficulties in defining domestic violence since other people see it as a norm and part of their culture. In Africa domestic violence may mean differently from western countries hence the need for education to create awareness among children while they are still young to detect such acts of family violence.

\section{HOW DOMESTIC VIOLENCE AFFECTS CHILDREN'S EDUCATION}

Literature reveals that children exposed to domestic violence suffer emotionally, psychologically, physically and socially (Lloyd, 2018; KaKanchiputu and Mwale, 2016; WHO, 2012). Pepler, Catallo \& Moore (2000) have stated that research on children exposed to family violence indicates that they are at risk for a wide range of both internalizing and externalizing behavior problems which vary according to their developmental stages. Maundeni (2000) reinforces the ideas above that the witnessing acts of domestic violence by children affected them directly or indirectly and caused them a lot of stress. Pepler, Catallo and Moore (2000) argue that; "internalizing problems are those which reflect the stresses that children endure such as : somatic complaints ( e.g. headaches), sleep disturbances, anxiety, fear of separation, social withdrawal , and depression." (p.39) whereas externalizing problems "refer to behaviors that children act out, such as : temper tantrums, impulsivity, hyperactivity, aggression, conflict with siblings and with peers, cruelty to animals, and bullying.(p.6) . There is very little evidence that suggests that there is a relationship between age and the effects of domestic violence, however, a study by Osofsky (1999) has drawn such relationships.

\section{Effects on Infants and Toddlers.}

Domestic violence is said to affect children differently according to their developmental stages. Osofsky (1999) research indicates that there are clear associations between exposure to violence, and emotional and behavioral problems. He further posits that infants and toddlers who witness violence either in their homes or community exhibit excessive irritability, 
immature behavior, sleep disturbances, emotional distress, fears of being alone, and regression in toileting and language. The argument is that exposure to trauma, especially violence in the family interferes with a child's normal development of trust, and later exploratory behaviors, which lead to the development of autonomy. The author further asserts that recent reports have noted the presence of symptoms in the young children very similar to post traumatic stress disorder in adults, including repeated re-experiencing of the traumatic event, avoidance, numbing of responsiveness, and increased arousal (Osofsky. 1999). The literature on infants and toddlers support the underlying assumption that children exposed to domestic violence exhibit internalizing and externalizing behaviors. Pepler, Catallo and Moore (200) augment these findings that children exposed to domestic violence had a lower sense of predictability.

\section{Effects on School Age Children}

According to Osofsky (1999) studies have reported that school age children who are exposed to family violence are affected in a similar way with those exposed to community violence. He further argues that:

Such children often show a greater frequency of internalizing (withdrawal, anxiety) and externalizing (aggressiveness, delinquency) behavior problems in comparison to children from non-violent families. Overall functioning, attitudes, social competence, and school performance are often affected negatively. In addition, studies show that as children get older, those who have been abused and neglected are more likely to perform poorly in school; to commit crime; and to experience emotional problems, sexual problems, and alcohol/ substance abuse (p. 37).

The above assertion clearly shows that children's exposure to domestic violence has long lasting implications on these children's lives as it impacts negatively on their academic, social and psychological aspects of their lives. The same factors have been observed that children exposed to family violence experience difficulties in paying attention and staying on task, that is they lack concentration, demonstrate poor academic achievement, problem solving skills and low levels of empathy. They also fall behind in their school work because of poor attendance, which may be attributable to fear for their mothers' safety when they leave home (Kanchiputu and Mwale, 2018 Pepler, Catallo and Moore, 2000); Carter, Weithorn and Behrman, 1999). The fear of children for their mothers is captured by Maundeni (2000) when a child described his experiences of extreme domestic violence and said;

He used a knife; his intention was to kill my mother. I was with my friends in the neighborhood, and all of a sudden, I saw my mother running out of the house covered with blood. I could not believe my eyes. My mother was hospitalized in intensive care unit for two weeks. During all that time, I prayed a lot. I had never prayed before in my life. I prayed time and again to God to help my mother to recover. Before the extreme violence he (my Father) inflicted on my mother, he used to beat her, but I never thought he could be so much violent to her (p.167).

The anecdote above clearly shows the stress and trauma that children suffer when there is violence that occurs in the family and one wonders how these children would cope in school with all these problems. Another scenario on the effects of domestic violence on children is captured by Mullender (2006) where a student was asked what affect her home life had on her and said:

A bad effect. At first, it was- I wouldn't leave my Mam, wouldn't leave her anywhere. I was around her all the time. And then, when I was 14, I used to stay out all the time... Anywhere, just to get out of the house... because I used to make situations worse by answering back (p.60). 
The two scenarios provide a clear picture of how domestic violence affects children physically, psychologically and emotionally. Maundeni (2001) opines that the effects of domestic violence on children do not necessarily end during childhood and sometimes spills into adulthood. This clearly indicates that exposure to domestic violence has long lasting effects on children. Mullender (2006) further argues that the impact domestic violence has depends on the individual child's level of understanding, personality, circumstances and coping strategies as well as the degree of support that they get. The author believes that there is no one syndrome that can be attributed to all children and that each child shows his or her distress in as wide a variety of ways as possible.

These responses can be age related, for example in younger children, one might experience sleep disturbances, eating disorder, unnatural quietness or clinginess. Pepler, Catallo \& Moore (2000) drawing from a theoretical perspective, cite Davies \& Cummings (1994) who have identified multiple risks experienced by children exposed to domestic violence that ; first, that children witness escalating problems from their father to their mother that can often be very traumatic for the children. Secondly, witnessing persistent marital conflict undermines the children's emotional security and their capacity to meet the demands of everyday-life. Thirdly, children exposed to family violence may suffer from chronic levels of arousal and deregulation. Consequently, children may use negative strategies in interactions with their parents, siblings and friends. The effects of domestic violence on children regardless of their age is detrimental to their wellbeing.

\section{Effects on Adolescents}

Osofsky (1999) contends that considerable research has been done on adolescents and violence as opposed to young children. Studies have shown that adolescents exposed to domestic violence tend to show high levels of aggression and acting out, accompanied by anxiety, behavioral problems, school problems, truancy, and revenge seeking. Various authors argue that even though exposure to domestic violence can have serious negative effects on children, they differ from one child to another. This is highly dependent on the family situation, environment and the child's own personality which may strengthen the child's ability to cope. It is believed that where there is domestic violence there is need for a loving parent to take care of the children and help them deal with the situation. However, it has been discovered that spouses who are victims of domestic violence often lose it and fail to protect their own children since they are also not in a stable state of mind. Therefore, it goes without saying that community structures and family structures like the extended family need to be conscientized on domestic violence as well such that they can be able to recognize it and offer support from the very beginning.

\section{PREVENTION STRATEGIES}

Wolfe \& Jaffe (1999) provide a public health model that can be used to inform the development of domestic violence strategies and opine that these can be divided into three categories. The three categories appear to be useful from an educational perspective; they are primary, secondary and tertiary. They opine that the primary prevention strategy involves efforts to reduce the incidence before it occurs, while secondary is aimed at decreasing the prevalence before escalating and tertiary attempts to minimize the course of the problem once it is diagnosed and problematic. The authors further categorize the preventative measures according to the children's age and these include infants and preschool age children, school age children and adolescents and high school age youths. The focus will be on the first three since they are applicable to school going children in my country 
Infants and School Age Children (0-5years).

According to Wolfe \& Jaffe (1999) primary and secondary prevention are necessary at this age because they focus on ensuring that the child gets a healthy start from the beginning which includes freedom from emotional, physical and sexual abuse. It is stated that these strategies begin with defining the principles of a healthy child-rearing environment with supportive and nurturing adults. To ensure that children are raised in a safe environment it is recommended that health workers should make home visits to check the security of the children after being born. This will enable policy makers to know the extent to which domestic violence is prevalent in their societies and plan in advance. This will also help diagnose serious cases before they escalate or even occur in order to save the child before any harm or exposure is done.

\section{School -Age Children (6-12 years)}

Schools, churches, and community centers have been seen to be traditional and as safe places for children exposed to domestic violence. Lloyd (2012) believe that schools are ideal places for introducing primary health care programs because that is where most of the children spend more time at attending school, much of their social learning occurs there and research has shown that social learning can play a role in the development of behaviors and attitudes that support domestic violence. Since teachers spend time with these children they can be used to help them in developing new ways of thinking and behaving. This idea is in tandem with Osofsky (1999) who believes that schools and teachers have an enormous potential for providing emotional support and nurturing for children exposed to domestic violence. Schools can even provide opportunities for children to benefit from the support of their peers which has been seen to be instrumental in reducing anxiety among children exposed to violence. He further argues that not only do churches provide support but also provide belief systems that have been shown to help children cope with trauma. The authors refer to these traditional forms of child protection as "safe havens" and see in them the potential to create awareness among children on domestic violence such that they can easily recognize it and report it in time.

According to Wolfe \& Jaffe (1999) one of the first programs to document efforts to prevent domestic violence by working with children was implemented in Minnesota Coalition for Battered Women. These programs were seen to be promising and focused on successful key elements such as:

identifying relationship violence as a form of societal problem; acknowledging that domestic violence is an abuse of power and control; creating a high enough level of trust so that children can disclose violence and teachers can make appropriate referrals; teaching safety skills about what to do when domestic violence occurs; and encouraging the development of social skills such as anger management and conflict resolution as alternatives to violence (p.139).

The argument is that children need to be exposed to issues of domestic violence at a tender age such that they have knowledge about it, develop relevant skills to deal with such issues and can report it as early as possible; this is viewed as one of the promising preventative strategies.

\section{Adolescents (13-18 years)}

Literature reveals that adolescence is a period of growth within children that is characterized by a lot of growth socially, cognitively and emotionally. It is believed that this is the time when they begin to develop understanding of the consequences of their behavior. It is at this stage that they rebel and no longer conform to parental views and opinions. Osofsky (1999) suggests that adolescence is the most opportune time to introduce primary prevention efforts 
in order to create awareness in ways in which violence in relationships can occur, and teach them healthy ways to form intimate relationships. Children are taught about choices and responsibilities. The author further cautions that teachers should send clear messages about personal responsibility and boundaries in a blame-free manner and not give lectures and warnings. According to Osofsky (1999) primary prevention programs provided in schools revolve around activities such as;

School auditorium presentations involving videotapes, plays, professional theater groups or speeches from domestic violence or teen dating violence survivors; classroom discussions facilitated by teachers or domestic violence services professionals; programs and curricula that encourage students to examine attitudes and behaviors that promote or violence; and peer support groups (p.140).

The primary prevention suggested by Osofsky tends to engage students in real life situations and discussions, and dialogue on what is going on around them. These activities have been seen to be productive among adolescents in a number of ways and it was observed that adolescents are receptive to school based prevention programs.

\section{CONCLUSION}

From the literature on this topic, it has become evident that domestic violence is a very intricate and complex issue because people exposed to it do not report such cases for a number of reasons. Some do not even recognize that it is a crime because of the cultural traits associated with it. Some people do not talk about it because they are ashamed to expose their families and also protective of their spouses. In other instances, it is not reported primarily because the perpetrator is the economic provider within his/her family, and there is fear that if reported he/ she will go to jail and the family will suffer. In other words domestic violence is linked to various factors which range from economic, power relations, prejudice, gender to culture and all these make it salient and appear to be acceptable. The strategies discussed in this paper have primarily focused on what schools can do to protect the children from a very early stage, and beyond the school, we suggest that different sectors of the society should devise other mechanisms to combat domestic violence and wage war against this pandemic.

\section{RECOMMENDATIONS}

The following recommendations are made for consideration by various stakeholders:

1. Children need to be conscientised on detecting perpetrators of domestic violence and ways of dealing with it.

2. Governments should develop legislation that tackles domestic violence.

3. Teachers should be trained to be able to identify and address issues of domestic violence among their students.

4. Schools should be made to serve as safe havens for children who have experienced domestic violence and psychologists be placed in such schools.

5. More research should be undertaken on dealing with domestic violence in educational settings such as schools.

\section{References}

American Bar Association. (1994). The impact of domestic violence on children: A report to the President of the American Bar Association. American Bar Association.

Anderson, K.L. (1997). Gender, status, and domestic violence: An integration of feminist and family violence approaches. Journal of marriage and the family, 5, 655-669.

Carter, S., Wethorn, L.A. and Behrman, R. E. (1999). Domestic violence and children: Analysis and Recommendations. The future of children, 9 (3). 4-20. 
Fantazzo, J. W. \& Mohr, W.K. (1999). Prevalence and effects of child exposure to domestic violence. The future of children, 9 (3) ,21-32.

Johnson, M.P. \& Ferraro, K.L. (2000). Research on violence in the 1990's: Making distinctions. Journal of marriage and the family , 62(4), 948-963.

Kanchiputu, P.G. and Mwale, M. (2016). Effects of domestic violence on children's education: The case of Mpemba, in Blantyre district (Malawi). Journal of Psychology of Abnormalities, 5(2), 1-5. DOI: 4172/2471-9900.1000152.

Lloyd, M. (2018). Domestic violence and education: Examining the impact od domestic violence on young children, children, and young people and the potential role of schools. Frontiers in Psychology Review, 9, 1-11. www.frontiersin.org. DOI: 10.3389/fpsyg.2018.02094.

Maundeni, T. (2001). The impact of parental separation and divorce on children: A Southern African Perspective. In A. Rwomire (Eds). African women and children: Crisis and response. (pp.27-48). Westport, Connecticut: Praeger.

Maundeni, T. (2000). Children living in violent families: Implications for social work in Botswana. Pula: Journal of African Studies, 14(2), 165-171.

Mullender, A. (2006). What children tell us: "He said he was going to kill our Mum." In C. Humphreys and N. Stanley (Eds). Domestic violence and child protection: Directions for good practice.( pp. 53-68). Philadelphia: Jessica Kingsley.

Okereke, G. O. (2002). Incidence of physical abuse in Nigeria: A pilot study. Research Review NS.,18 (2),39-51.

Osofsky, J. D. (1999). The impact of violence on children. The future of children, 9 (3), 33-49.

Pepler, D. J., Catallo, R. \& Moore, T. E. (2000) Consider the children: Research informing interventions for children exposed to domestic violence. In R. A. Geffner, P. G. Jaffe, and M. Sudermann (Eds). Children exposed to domestic violence: Current issues in research, intervention, prevention, and policy development. (pp. 37-58). New York: Harworth Press, Inc.

Republic of Botswana. (2012). Gender based violence indicators study in Botswana. Gaborone: Women’s Affairs Department. www.gov.bw

Wolfe, D. A. \& Jaffe, P. G. (1999). Emerging strategies in the prevention of domestic violence. The Future of Children, 9 (3), 133-144.

World Health Organization. (2012). Intimate partner violence. http://www.who.int/about/licensing/copyright-form/en/index.html 